\title{
Cadherins are regulated by Ep-CAM via phosphaditylinositol-3 kinase
}

\author{
Manon J. Winter · Vincenzo Cirulli • \\ Inge H. Briaire-de Bruijn · Sergey V. Litvinov
}

Received: 11 August 2006/ Accepted: 19 January 2007 / Published online: 14 February 2007

(C) Springer Science+Business Media B.V. 2007

\begin{abstract}
The cross-signaling between (cell) adhesion molecules is nowadays a well-accepted phenomenon and includes orchestrated cellular changes and changes in the microenvironment. For example, Ep-CAM is an epithelial adhesion molecule that prevails in active proliferating tissue and is suppressed in a more differentiated state of the cell. E-cadherin adhesion complexes are typical for the advanced and terminal differentiated cell status. During normal proliferation, E-cadherin is not suppressed. We have demonstrated the effect of overexpression of Ep-CAM on E-cadherin, which probably affects the connection of cadherins and F-actin. Phosphatidylinositol 3-kinase $(\mathrm{Pi} 3 \mathrm{~K})$ participates in various regulating mechanisms, for example in signaling to nuclei, vesicle transport, and cytoskeletal rearrangements. The effect of Ep-CAM on E-cadherin mediated junctions as well as the involvement of $\mathrm{Pi} 3 \mathrm{~K}$ in regulating adherens junctions, led us to investigate the potential interaction between Pi3K and Ep-CAM. Introduction of Ep-CAM in the epithelial cells caused abrogation of $\mathrm{N}$-cadherin mediated cell-cell adhesion, which could be inhibited by Pi3K inhibitor LY294002. Moreover, the Pi3K subunit p85 was precipitated with Ep-CAM from cell lysates, and this complex showed kinase activity. The
\end{abstract}

M. J. Winter $(\bowtie)$ I I. H. Briaire-de Bruijn

Department of Pathology, Leiden University Medical

Center, P.O. Box 9600, 2300 RC Leiden, The Netherlands

e-mail: winterm1@wyeth.com

V. Cirulli

Whittier Institute, UCSD, La Jolla, CA, USA

S. V. Litvinov

Pickcell Laboratories, Amsterdam, The Netherlands
Pi3K activity shuttled from N-cadherin to Ep-CAM.From our results, we conclude that Ep-CAM cross signaling with $\mathrm{N}$-cadherin involves $\mathrm{Pi} 3 \mathrm{~K}$, resulting in the abrogation of the cadherin adhesion complexes in epithelial cells.

Keywords Differentiation - Cadherin - Ep-CAM . Pi3K · Cell adhesion

\section{Introduction}

The maintenance of tissue integrity is a complex and dynamic process, requiring a balance between a variety of signaling pathways. Responding to external signals requires many secondary pathways within the cells in order to respond accurately and in a coordinated fashion [1]. Disturbances in these pathways could lead to dramatic dysfunctioning of the cell, for example carcinogenesis. Diagnosis of early pathological development is very difficult. First, insight and understanding of the disturbed regulating pathways is required.

Ep-CAM is an adhesion molecule that is expressed in all simple, pseudostratified epithelia, but not in mature squamous epithelia. It is strongly associated with cellular proliferation in vitro as well as in vivo [2]. Tumors originating from epithelial cells over-express or express de novo Ep-CAM. De novo expression is already detected in the precursors of carcinomas of squamous epithelium, where Ep-CAM expression serves as an early marker $[2,3]$.

Phosphatidylinositol 3 kinase ( $\mathrm{Pi} 3 \mathrm{~K}$ ) is an ubiquitous enzyme involved in many intracellular signal transduction pathways. It participates in various regulating mechanisms, for example in cytoplasmic- to 
nuclear signaling, vesicle transport, cytoskeletal rearrangements and CD28-B7 outside-inside signaling $[1,4,5]$. Important target molecules are the small Rho GTPases, which are involved in cytoskeletal organization. Cytoskeleton (re-) arrangement results in signaling to integrins and cadherins. Ras/GTP, activated by growth factors, promotes Pi3K activity and thereby inhibits apoptosis [6]. The Pi3K protein consists of an $85 \mathrm{kDa}$ and a $110-\mathrm{kDa}$ subunit. The p85 subunit is recognized as being the regulatory subunit, while the p110 subunit employs the actual kinase activity that converts plasma membrane lipid $\mathrm{PIP}_{2}$ into $\mathrm{PIP}_{3}$.

E-cadherin adhesion complexes are typical for the advanced and terminal differentiated cell status. The Ep-CAM expression in epithelia inversely correlates with cadherin-mediated junction complexes. In vitro studies demonstrated that cadherin-mediated adhesions are abrogated upon Ep-CAM introduction, probably by affecting the connection between cadherin adhesion complexes and $\mathrm{F}$-actin $[7,8]$. This results in reversing the E-cadherin-induced epithelial phenotype of L-cells into a mesenchymal phenotype $[2,7]$. Our in vivo studies revealed a regulated expression of Ep-CAM in pancreatic islet ontogeny [9]. During maturation of the pancreatic islet-like cells, Ep-CAM expression decreases and simultaneously the (endocrine) differentiation increases. Pi3K was demonstrated to negatively regulate the differentiation in human fetal pancreatic cells [10]. In cells expressing cadherins, cytoskeletal rearrangements are observed upon Ep-CAM introduction [8]. These rearrangements may be the result of involvement of small GTPases or Pi3K.

The results described here, show that signaling between Ep-CAM and cadherins involves activation of Pi3K.

\section{Material and methods}

Cells, constructs and cell culture

Human breast epithelial cells HBL100 (clone HCA, kindly provided by Dr. Hilkens, The Netherlands Cancer Institute, Amsterdam. The Netherlands) were transiently transfected with full length human Ep-CAM cDNA that was subcloned into the pMep4 vector (Invitrogen BV., Leek, the Netherlands), using the HindIII/Bgl restriction sites [7]. The construct is placed under the control of the methallothionin promotor, which can be induced by divalent heavy metal ions (e.g., $\mathrm{Cd}^{2+}$ or $\mathrm{Zn}^{2+}$ ). This allows a stepwise expression of Ep-CAM. Transfection was performed with Fugene ${ }^{\mathrm{TM}} 6$ Transfection Reagent (Boehringer Mannheim,
Mannheim, Germany) and selected by $1 \mathrm{mg} / \mathrm{ml} \mathrm{Hy}-$ gromycin B (Boehringer Mannheim), as described earlier [7].

For total-Akt and phospho-Akt blots, cells were serum starved overnight, and re-administered $2 \%$ fetal bovine serum during $8 \mathrm{~h}$ in the presence or absence of $\mathrm{CdCl}_{2}$ or $25 \mu \mathrm{M} \mathrm{LY} 294004$ (LY) before lysing. $\mathrm{CdCl}_{2}$ induces the transcription of Ep-CAM, LY294004 inhibits the Pi3K activity.

Antibodies and chemicals

The monoclonal antibodies anti-N-cadherin (clone \#32), anti-E-cadherin (clone \#36), anti- $\alpha$-catenin, anti$\beta$-catenin, and anti-p85 were obtained from Transduction Laboratories (Lexington, KY). Polyclonal antibodies against total-Akt and phospho-Akt are from Cell Signaling Tech. (Beverly, MA). The monoclonal antibody 323/A3, directed against Ep-CAM, was kindly provided by Centocor B.V., the Netherlands. Secondary antibodies for immunoblotting were labeled with HRP (Tansduction Laboratories) and for immunofluorescent stainings isotype specific antibodies were labeled with either Alexa-488, Alexa-546 (Molecular Probes Europe BV, Leiden, the Netherlands), or Cy-5 (PickCell, Amsterdam, the Netherlands).

For immunoprecipitations, Protein G Sepharose beads from Amersham were labeled (Amersham Pharmacia Biotech, Buckinhamshire, UK). At least $16 \mathrm{~h}$ prior to fixation or lysis of cells, $25 \mu \mathrm{M}$ LY294002 (Biomol Research Laboratories Inc., Plymouth Meeting, PA) was added to the culture medium to inhibit Pi3K. Ep-CAM expression in transfected cells was enhanced by overnight incubation of 10 or $25 \mu \mathrm{M} \mathrm{CdCl}_{2}$.

Immunoprecipitation

Cells were grown to $80 \%$ density and overnight induced $\left(25 \mu \mathrm{M} \mathrm{CdCl}_{2}\right)$. The procedure was followed as described previously [11], using TritonX100, $\beta$-octyl glucopyranoside, or CHAPS as detergents.

Detergent extraction, total lysates, and Western Blot analysis

For extraction, overnight induced $\left(25 \mu \mathrm{M} \mathrm{CdCl}_{2}\right)$ cell cultures were rinsed with ice-cold $1 \mathrm{mM} \mathrm{CaCl}_{2} / 1 \mathrm{mM}$ $\mathrm{MgCl}_{2}$ in PBS and extracted with $1 \%$ TritonX-100 or $50 \mathrm{mM}$ CHAPs in extraction buffer as described previously [11]. The samples were collected and spun down $\left(15^{\prime}, 15,000 \mathrm{rpm}\right)$. The detergent-insoluble fraction of cells was separated from supernatant (the detergentsoluble fraction), and further lysed in hot $1 \%$ SDS/ 
$10 \mathrm{mM}$ EDTA. For total lysates, the cells were lysed in hot lysis buffer immediately after rinsing off the culture medium with ice-cold $1 \mathrm{mM} \mathrm{CaCl}_{2} / 1 \mathrm{mM} \mathrm{MgCl}_{2}$ in PBS.

Protein concentrations in homogeneous lysates were measured using Bio-Rad $D_{C}$ Protein Assay (Bio-Rad Lab., Hercules, CA). For all Western blots, $10 \mu \mathrm{g}$ of homogenous lysate was loaded per lane of the $10 \%$ SDS-gel, representing equal protein load per lane while avoiding control stainings [7, 8, 11]. Proteins were separated by SDS/PAGE, and transferred electrophoretically onto PVDF-membrane (Millipore, Bedford, MA). The membrane was stained as described previously [7]. The blots were visualized using the ECL detection substrate (Amersham).

\section{Pi3K activity assay}

The Pi3K activity of anti-p85 immunoprecipitates was analyzed as described previously [10].

Briefly, aliquots of cell lysates normalized for protein content were incubated with anti-Ep-CAM antibodies and absorbed onto protein A-Sepharose and washed. The reaction was carried out for $10 \mathrm{~min}$ in a buffer that also contained $10 \mu \mathrm{g}$ of PI (Avanti Polar Lipids, Alabaster, AL) and $10 \mu \mathrm{Ci}[\gamma-32 \mathrm{P}] \mathrm{ATP}$ $(6,000 \mathrm{Ci} / \mathrm{mmol}$; DuPont/NEN, Wilmington, DE). After the incubation, the reaction was stopped with methanol plus $2.4 \mathrm{~N} \mathrm{HCl}(1: 1, \mathrm{vol} / \mathrm{vol})$, and lipids were extracted, and analyzed by thin-layer chromatography.

\section{Confocal microscopy}

Cells at $80 \%$ density and overnight induced $(25 \mu \mathrm{M}$ $\mathrm{CdCl}_{2}$ ) were washed with ice-cold $1 \mathrm{mM} \mathrm{CaCl} / 1 \mathrm{mM}$ $\mathrm{MgCl}_{2}$ in $\mathrm{PBS}$, and fixed during 15 min with $-20^{\circ} \mathrm{C}$ methanol and allowed to dry. Cells were blocked with $5 \%$ skim milk in PBS, the primary monoclonal antibody was incubated for $1 \mathrm{~h}$, followed by an hour incubation with a fluorochrome labeled secondary antibody.

For actin staining, cells were fixed for $1 \mathrm{~h}$ in $4 \%$ paraformaldehyde, permeabilized with $0.3 \%$ TritonX100 [11], and incubated with TRITC-labeled phalloidin (Sigma-Aldrich Chemie Gmbh, Steinheim, Germany). After drying, cells were mounted and examined by confocal microscopy (Zeiss LSM 510, Jena, Germany).

\section{Results}

Pi3K switches between Ep-CAM and N-cadherin

The breast epithelial cell line HBL100 was transiently transfected with pMep4 plasmids containing either wt Ep-CAM cDNA, HME, or empty vector, HMC. cDNA transcription under control of the methallothionin promotor can be enhanced by incubation with divalent cations. This enables a 3-step increase in expression level by (1) the mock transfectant (promotor only, HMC), (2) transfectant with 'normal' transcription (HME), and (3) transfectant with enhanced expression (HME $+25 \mu \mathrm{M} \mathrm{CdCl}{ }_{2}$ ) [11]. Cell surface expression of Ep-CAM was measured by flow cytometry analysis (Fig. 1a).

Cross-signaling between E-cadherin and Ep-CAM has previously been demonstrated to involve the connection between cadherin-mediated cell-cell junctions and F-actin $[8,11]$. Cytoskeletal rearrangements are observed upon Ep-CAM introduction in cells expressing N-cadherin (Fig. 1b). In HME cells, the actin cytoskeleton is concentrated in the cortical area, in contrast to HMC cells where more fibers can be found.

Co-immunoprecipitation for N-cadherin (Fig. 1c) or Ep-CAM showed that the Pi3K regulatory subunit, p85, shuttles between cadherin and Ep-CAM; most p85 is complexed with $\mathrm{N}$-cadherin in HMC cells, while in Ep-CAM expressing HME cells, the precipitation complex with $\mathrm{N}$-cadherin has 'lost' most of the p85 molecules. Immunoprecipitation with Ep-CAM only precipitated molecules in the Ep-CAM expressing HME cells, where p85 has appeared in the complex. Interestingly, performing the immunoprecipitation for Ep-CAM on high $(95 \%)$ or low $(15 \%)$ density cell cultures, p85 and Ep-CAM only co-precipitated in HME cells from high density cultures (Fig. 1d).

\section{Effect of Ep-CAM on N-cadherin can be blocked} by LY

Western blotting of total lysates of HMC, HME, and $\mathrm{HME}+\mathrm{CdCl}_{2}$ cells showed that the amount of the regulatory subunit of $\mathrm{Pi} 3 \mathrm{~K}$, p85, had not changed in these cells (Fig. 2a). Pi3K inhibitor LY294004 (LY) incubation resulted in a slight decrease of total $\mathrm{N}$-cadherin in both $\mathrm{HMC}+\mathrm{CdCl}_{2}$ and $\mathrm{HME}+$ $\mathrm{CdCl}_{2}$. Ep-CAM expression was strongly upregulated in $\mathrm{HME}+\mathrm{CdCl}_{2}$ cells, but upon LY incubation the total expression of Ep-CAM had decreased.

Immunofluorescent staining for $\mathrm{N}$-cadherin and $\alpha$-catenin showed that these molecules were present at the cell-cell boundaries in HMC, most likely in cadherin-mediated adhesion complexes (Fig. 2b). The cadherin-mediated adhesion complex had turned more diffuse in HME cells, where Ep-CAM was expressed. Upon LY incubation, the $\mathrm{N}$-cadherin and $\alpha$-catenin re-located to the cell plasma membrane, showing the 
A

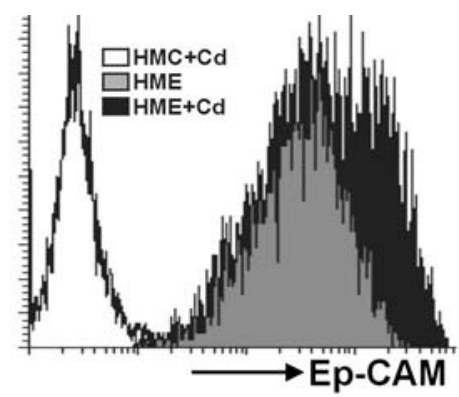

B

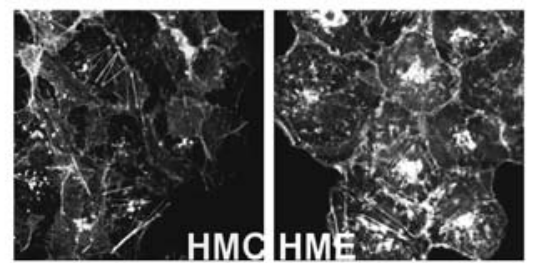

C
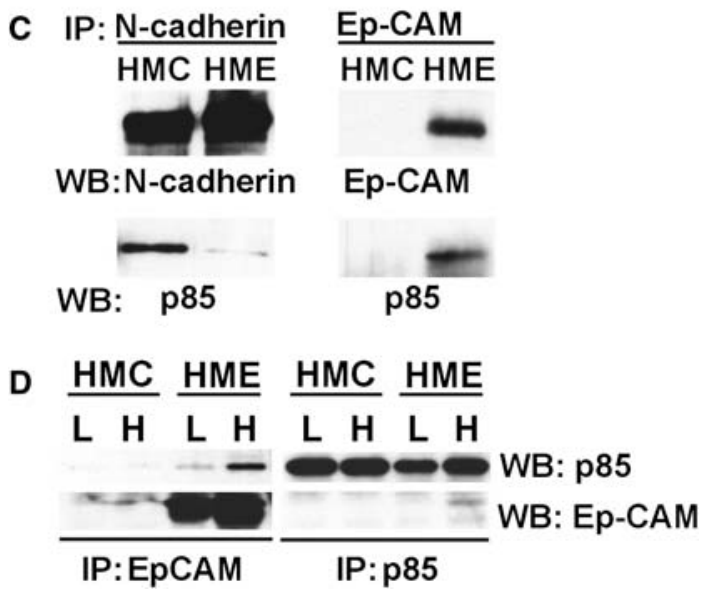

Fig. 1 Expression of Ep-CAM in HbL100 cells. (a) Flow cytometry analysis of mock (HMC) and Ep-CAM transfected HbL100 cells (HME). The mock-transfected cells, HMC, did not express Ep-CAM at the cell surface (white). The Ep-CAM expression was detected in HME (gray), which was increased upon overnight incubation with $\mathrm{CdCl}_{2}$ (black). (b) F-actin staining of overnight $\mathrm{CdCl}_{2}$ induced $\mathrm{HMC}$ and $\mathrm{HME}$ cells, using TRITC-conjugated phalloidin. In HME cells, the actin cytoskeleton is concentrated in the cortical area, in contrast to HMC cells, where more fibers can be found. (c) $10 \mu \mathrm{g}$ of homogenous lysate was loaded per lane of the $10 \%$ SDS-gel, representing equal protein load per lane. Western blotting total lysates of HMC and HME and probed for N-cadherin, Ep-CAM and the Pi3K subunit p85. Cell cultures were lysed after overnight incubation with $\mathrm{CdCl}_{2}$ and LY. P85 shuttled between $\mathrm{N}$-cadherin and Ep-CAM. In HMC cells, p85 precipitated with $\mathrm{N}$-cadherin. Upon Ep-CAM introduction, HME cells, p85 now precipitated with Ep-CAM. (d) Immunoprecipitation for Ep-CAM and p85 on high $(\mathrm{H})$ or low $(\mathrm{L})$ density cell cultures, and Western blotted for Ep-CAM and p85. P85 and Ep-CAM only co-precipitated in HME cells from high densed cultures

'spike'-like staining pattern in $\mathrm{HME}+\mathrm{CdCl}_{2} / \mathrm{LY}$, while Ep-CAM had left the cell-cell boundaries.

Ep-CAM transcription and expression in cell cultures $(80 \%$ monolayer $)$ were induced with $10 \mu \mathrm{M}$
$\mathrm{CdCl}_{2}$. Cells were subsequently incubated with $25 \mu \mathrm{M}$ Pi3K inhibitor LY. Cells were CHAPS detergent extracted before lysing them, allowing discrimination between the soluble and insoluble proteins. Figure 2c shows the soluble fraction that represents the cadherins and catenins that are not bound to the cytoskeleton, i.e., not complexed in cell-cell adherens junctions. As a lysate, the soluble fractions of CHAPS extracted cells (i.e., non-bound to F-actin) were Western blotted and stained for $\mathrm{N}$-cadherin, p85, Ep-CAM, $\beta$-catenin, and $\alpha$ catenin. Per lane, $10 \mu \mathrm{g}$ of each homogenous lysate was loaded, representing equal protein load per lane while avoiding control stainings. Figure $2 \mathrm{c}$ demonstrates that the $\mathrm{N}$-cadherin, $\beta$-catenin, and $\alpha$-catenin levels had increased upon $\mathrm{CdCl}_{2}$ enhanced Ep-CAM (Fig. 2c). Only the soluble fraction of cadherins and catenins increased while the total amount remained unchanged (not shown, see [7]). The increased proteins in the soluble fraction indicates that more cadherin junctional complexes are disconnected from the cytoskeleton upon Ep-CAM introduction, and not the result of increased cadherin and catenin expression (the total amount of cadherins and cadherins did not change). By blocking Pi3K activity by LY incubation, levels of soluble $\mathrm{N}$-cadherin, $\beta$-catenin and $\alpha$-catenin decreased, although Ep-CAM expression levels were still high.

It is plausible that Ep-CAM, which is associated with proliferation both in vitro and in vivo [2], regulates epithelial cell differentiation via the Pi3K/Akt signaling pathway. To validate the active state of $\mathrm{Pi} 3 \mathrm{~K}$, the level of phosphorylation of Pi3K substrate, Akt, was measured. Serum-starved HMC and HME were Western-blotted for total-Akt and phosphorylated-Akt (P-Akt, Fig. 2d). Total lysates of both HMC and HME hardly showed any P-Akt. Addition of $2 \%$ serum had activated $\mathrm{Pi} 3 \mathrm{~K}$, as can be concluded from the increase in P-Akt per $10 \mu \mathrm{g}$ in both $\mathrm{HMC}$ and $\mathrm{HME}$. Incubation with $\mathrm{CdCl}_{2}$, which enhanced the expression of Ep-CAM in HME, increased the level of P-Akt in HME, as compared to serum-induced HME cells. This increase of Pi3K activity could partially be blocked by LY, although the levels of P-Akt in HME appeared to have decreased more than in HMC. Since equal protein concentrations were blotted and the total levels of Akt did not change, this demonstrates Pi3K activity as a result of Ep-CAM expression.

Pi3K is associated with Ep-CAM

Immunoprecipitations with the Pi3K regulatory subunit, p85, using different cell lysing buffers, 


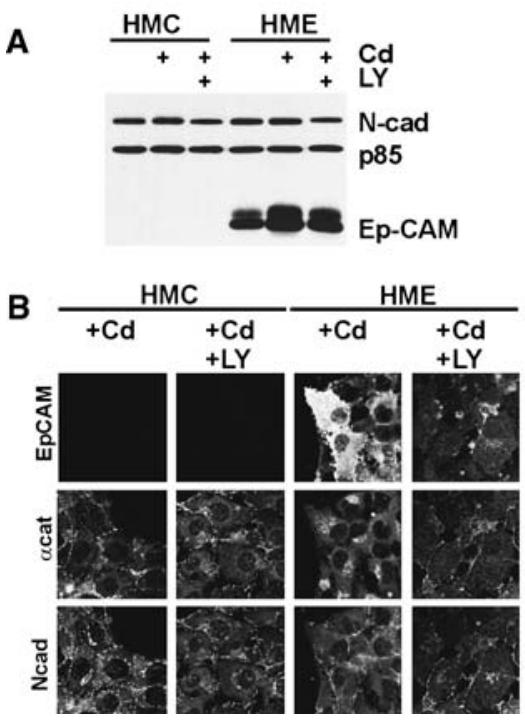

Fig. 2 Abrogation of N-cadherin-mediated adhesions. (a) $10 \mu \mathrm{g}$ of homogenous lysate was loaded per lane of the $10 \%$ SDS-gel, representing equal protein load per lane. Western blotting of total lysates of CHAPS extracted HMC and HME cell cultures after $\mathrm{CdCl}_{2}$ and/or LY incubation, stained for N-cadherin, EpCAM and p85. In HME cells, LY incubation decreased the high Ep-CAM expression levels. (b) Immunofluorescent staining of HMC and HME for Ep-CAM, $\alpha$-catenin and N-cadherin after incubation with $\mathrm{CdCl}_{2}$ and LY. Ep-CAM expression in HME cells, abrogated the presence of $\mathrm{N}$-cadherin and $\alpha$-catenin at the cell-cell boundaries. In these same HME cell, inhibition of Pi3K by LY, resulted in re-location of $\mathrm{N}$-cadherin and $\alpha$-catenin at the cell-cell boundaries. (c) Western blotting of the soluble fraction of CHAPS extracted HMC and HME cell cultures after $\mathrm{CdCl}_{2}$ and/ or LY incubation, stained for N-cadherin, Ep-CAM, Pi3K

demonstrated that Ep-CAM is present in the precipitated complex (Fig. 3a).

It remained unclear whether this Ep-CAM/p85 complex included the Pi3K subunit p110 that employes the kinase activity. Therefore, a kinase activity assay was performed (Fig. 3b): The Ep-CAM/p85 complex was precipitated with the anti-Ep-CAM antibody and subsequently incubated with 32-P labeled ATP and PI. If the Ep-CAM/p85 complex would have kinase activity, this would result in the formation of $\mathrm{PI}_{3} \mathrm{P}$, while no $\mathrm{PI}_{3} \mathrm{P}$ was formed when precipitated with a control antibody.

\section{Discussion}

Cross-signaling between cadherins and other adhesion molecules is a common phenomenon [1]. Ep-CAM expression is associated with proliferation and inhibits epithelial differentiation $[7,9,12]$. In a previous study we showed that the communication between Ep-CAM and E-cadherin does not involve the regulatory mole-

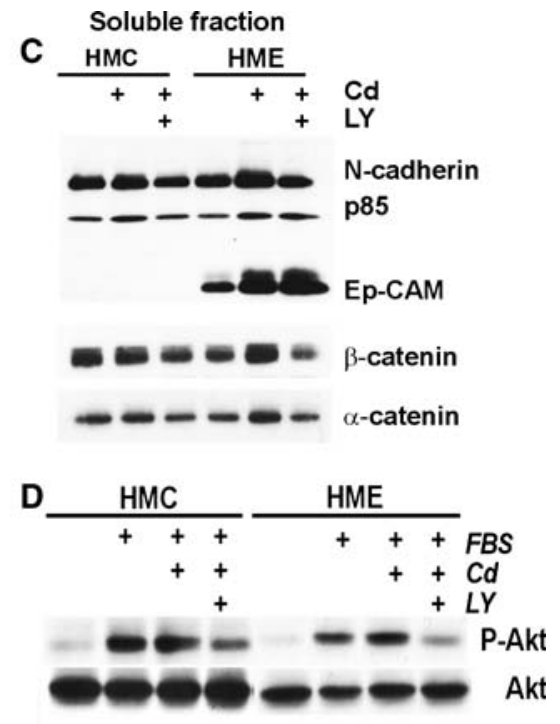

subunit $\mathrm{p} 85, \beta$-catenin, and $\alpha$-catenin. The soluble fraction represents the cadherins and catenins that are not bound the cytoskeleton, i.e., not complexed in cell-cell adherens junctions. In HME cells, the soluble fraction of Ep-CAM increased upon LY incubation, while the soluble fractions of $\beta$ - and $\alpha$-catenin decreased. (d) Western blotting for total-Akt and phosphorylated-Akt (P-Akt) in HMC and HME total lysates after serum starvation and subsequent addition of FBS, $\mathrm{CdCL}_{2}$, and LY. In serum starved HMC and HME cells (first lanes of both) there was hardly any phosphorylated Akt detectable. Addition of FBS induced the p-Akt in HMC and HME, and upon increased expression of Ep-CAM (Cd induced), more P-Akt was detected in HME. This increase in P-Akt in HME was only partially blocked by LY, although the levels of P-Akt in HME appeared to be decreased more than in HMC

cule $\beta$-catenin [8]. Ep-CAM abrogated the E-cadherinmediated adhesion complex by disrupting the connection between $\alpha$-catenin and the actin cytoskeleton. An identical response to Ep-CAM introduction was found for N-cadherin [11]. Since this is not the result of a physical interaction, one or more other molecules have to be involved [8]. In the present study, we show that cross-signaling between Ep-CAM and classical cadherins involves the Pi3K signaling pathway. This was already suggested by the fact that $\mathrm{Rac} / \mathrm{Pi} 3 \mathrm{~K}$ activation induced disassembly of cadherin-mediated adhesions. The same findings have been described for Ep-CAM expression [13-15]. As previously described, both Ep-CAM and p85, the regulatory subunit of Pi3K, are capable to bind $\alpha$-actinin, although under different conditions [11, 16]. Our results demonstrating co-precipitation of Ep-CAM and p85 suggest for the first time that these molecules do interact. Furthermore, our results indicate that this complex has kinase activity. Both Ep-CAM and E-cadherin mediatedadhesions are localized at the basal-lateral membrane and mediate cell-cell contacts by themselves. Ep-CAM 


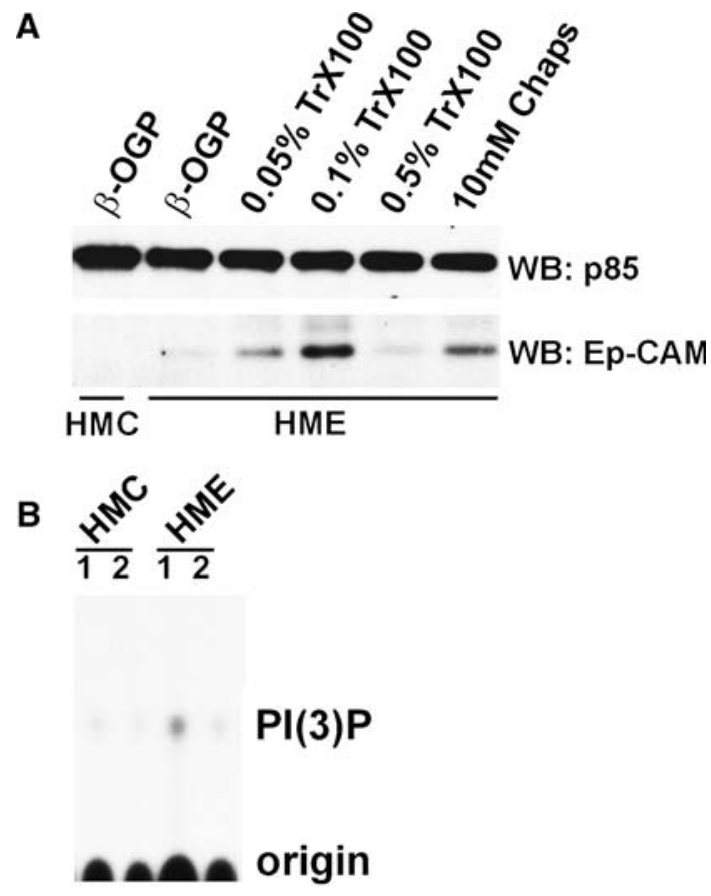

Fig. 3 Ep-CAM and Pi3K in signaling. (a) $10 \mu \mathrm{g}$ of homogenous lysate was loaded per lane of the $10 \%$ SDS-gel, representing equal protein load per lane. Immunoprecipitation with Pi3K subunit $\mathrm{p} 85$ in lysates of several detergents, and blotted for $\mathrm{p} 85$ and Ep-CAM. OGP: $\beta$-octyl glucopyranoside. Ep-CAM is present in the precipitated complex. (b) Kinase activity assay for precipitates from $\mathrm{HMC}$ and $\mathrm{HME}$. The formation of $\mathrm{PI}_{3} \mathrm{P}$ in HME cells is the result of kinase activity in the Ep-CAM/p85 complex (lane 1; precipitate with anti Ep-CAM antibody), while no $\mathrm{PIP}_{3}$ was formed when precipitated with a control antibody (lane 2; anti-IgG)

also regulates cadherin-mediated adhesions, which might be modulated by shuttling of p85. Nelson and Chen reported that Pi3K activity is required for cellcell contact induced proliferation, which is in line with our results [17].

Pi3K activation promotes the cell entering the $\mathrm{S}$ phase of the cell cycle and Pi3K activation promotes cellular changes that are characteristic of premalignant transformation [18]. Ep-CAM is associated with proliferation both in vitro and in vivo and de novo expression was demonstrated in adult squamous carcinomas $[2,3]$. Akt has been shown to repress transcription of the E-cadherin gene. Second, cells expressing constitutively active Akt produce the transcription factor Snail that represses the expression of the E-cadherin gene [19]. Ep-CAM and E-cadherin expression are reversely correlated in epithelial cells and forced expression results in abrogation of $\mathrm{E}$ cadherin-mediated adhesions $[2,8]$. Therefore, it is not unlikely that Ep-CAM is involved in de Pi3K/Akt signaling pathway regulating epithelial cells upon external signals.
Signaling pathways downstream from $\mathrm{Pi} 3 \mathrm{~K}$ are implicated in cell dissociation and scattering in MDCK cells [20]. Furthermore, forced Ep-CAM expression in transgenic mice induced budding and secondary branching of the mammary glandular tree in virgin females [21]. MAPK and Pi3K activation by Ras is required for HGF/SF-induced adherens junction disassembly in MDCK cells [14, 20], and Pi3K activity promotes invasiveness in epithelial breast cells [22, 23]. Ep-CAM promotes invasiveness in Ep-CAM transfected HBL100 cells in matrigel (our unpublished data) and the relationship with the small GTPases in this process is under investigation.

Both Ep-CAM and p85, the regulatory subunit of $\mathrm{Pi} 3 \mathrm{~K}$, can bind $\alpha$-actinin, although under different experimental conditions [11, 16]. Recently, p85 was found to bind to a specific domain in membrane-bound ezrin, a plasma membrane-microfilament linker. Moreover, Gautreau and co-workers stated that survival signaling by ezrin is regulated through the $\mathrm{Pi} 3 \mathrm{~K} /$ Akt pathway [24]. ICAM-2 clustering induced ezrin tyrosine phosphorylation and enhanced threonine phosphorylation, concomitant with an increase in $\mathrm{p} 85 /$ Pi3K association in Jurkat $\mathrm{T}$ cells [25]. It is possible that in the complex of Ep-CAM and Pi3K, ezrin is also present sustaining a survival in cells that have downregulated E-cadherin mediated adhesion.

Potempa and Ridley found that the disassembly of cadherin junctional complexes in migrating $\mathrm{HGF} / \mathrm{SF}$ stimulated MDCK cells could be blocked by Pi3K inhibitors LY or PD98059 incubation as well as by micro-injected dominant negative MAPKK1 [14]. However, activation of Pi3K or p42/p44 MAPK was not sufficient to induce a disruption of adherens junctions. Wennström and Downward reported similar results in EGF stimulated COS-7 cells [26]. They postulated that the ability of LY to inhibit the EGF-R/ Ras/Raf/MEK pathway is due to basal Pi3K activity that contributes to Ras activation upon weak stimuli (e.g., low concentration of EGF) [26]. Pi3K activity downstream from Ras can only be induced by high concentration of EGF induced Ras or oncogenic Ras. The basal Pi3K activity may be provided by Ep-CAM expression.

A role for Ep-CAM as a morphoregulatory molecule becomes more plausible. Ep-CAM expression implicates proliferation and differentiation, as previously demonstrated $[3,7,9,12]$. Pi3K physically interacts with activated growth receptors, transducing survival signals. The signaling between Ep-CAM and Pi3K has its consequences for cellular motility and/or invasive capacity. Disturbed regulation of these molecules may result in increased metastatic capacity, 
possibly by affecting the small Rho GTPases and thereby inducing cytoskeletal rearrangements.

It can be concluded from the results described here that Pi3K can act downstream from Ep-CAM. The question of a potential feedback loop from Ep-CAM to Pi3K remains to be studied. Our first experiments do not eliminate the possibility. E.g., expression of Ep-CAM deletion mutants incapable of adhesion, results in altered p85 expression levels (unpublished observations). These preliminary observations would be in line with conclusions from other research groups. For example, Akt, acting downstream of Pi3K, is activated by the formation of E-cadherin-mediated junctions [27], while formation of E-cadherin-mediated junctions was demonstrated to be inversely correlated to Ep-CAM expression [7]. Although the mechanism of a putative regulation loop needs further investigation, such a mechanism may explain the observations of both a de novo Ep-CAM expression in cervical carcinoma [2] and the amplification of the gene encoding for $\mathrm{Pi} 3 \mathrm{~K}$ subunit $\mathrm{p} 110 \alpha$ in cervical and ovarian carcinomas $[28,29]$.

The experiments reported here demonstrate that Pi3K can act downstream from Ep-CAM. Abnormal signaling between these molecules may play a crucial role in the biology of some epithelial tumors.

Acknowledgments V. Cirulli was supported by NIH grants DK55183 and DK63443, JDRF Grants \#1-2004-13, and \#1-20051084, and a Network Grant from The Larry L. Hillblom Foundation.

\section{References}

1. Alahari SK, Reddig PJ, Juliano RL (2002) Biological aspects of signal transduction by cell adhesion receptors. Int Rev Cytol 220:145-184

2. Litvinov SV, van Driel W, van Rhijn CM, Bakker HAM, van Krieken H, Fleuren GJ, Warnaar SO (1996) Expression of Ep-CAM in cervical squamous epithelia correlates with an increased proliferation and the disappearance of markers for terminal differentiation. Am J Pathol 148:865-875

3. Winter MJ, Nagtegaal ID, van Krieken H, Litvinov SV (2003) The epithelial cell adhesion molecule Ep-CAM as a morphoregulatory molecule is a tool in surgical pathology. Am J Pathol 163:2139-2148

4. Fukui Y, Ihara S, Nagata S (1998) Downstream of phosphatidylinositol-3 kinase, a multifunctional signaling molecule, and its regulation in cell responses. $\mathrm{J}$ Biochem 124:1-7

5. Cefai D, Cai YC, Hu H, Rudd C (1996) CD28 co-stimulatory regimes differ in their dependence on Pi3K: common co-signals by CD80 and CD86. Int Immunol 8:16091616

6. Marshall CJ (1996) Ras effectors. Curr Opin Cell Biol 8:197-204

7. Litvinov SV, Balzar M, Winter MJ, Bakker HAM, Briaire-de Bruijn IH, Prins FA, Fleuren GJ, Warnaar SO (1997) Epi- thelial adhesion molecule Ep-CAM modulates cell-cell interactions mediated by classic cadherins. J Cell Biol 139:1337-1348

8. Winter MJ, Nagelkerken B, Mertens AEE, Rees-Bakker HAM, Briaire-de Bruijn IH, Litvinov SV (2003) Expression of Ep-CAM shifts the state of cadherin-mediated adhesions from strong to weak. Exp Cell Res 285:50-58

9. Cirulli V, Crisa L, Beatti GM, Mally MI, Lopez AD, Fannon A, Ptasznik A, Inverardi L, Ricordi C, Deerinck T, Reisfeld RA, Hayek A (1998) KSA antigen Ep-CAM mediates cell-cell adhesion of pancreatic epithelial cells: morphoregulatory roles in pancreatic islet development. J Cell Biol 140:1519-1534

10. Ptasznik A, Beatti GM, Mally MI, Cirulli V, Lopez AD, Hayek A (1997) Phosphatidylinositol 3-kinase is a negative regulator of cellular differentiation. J Cell Biol 137:11271136

11. Balzar M, Bakker HAM, Briaire-de Bruijn IH, Fleuren GJ, Warnaar SO, Litvinov SV (1998) Cytoplasmic tail regulates the intercellular adhesion function of the epithelial adhesion molecule. Mol Cell Biol 18:4833-4843

12. De Boer CJ, van Krieken H, Janssen-van Rhijn CM, Litvinov SV (1999) Expression of Ep-CAM in normal, regenerating, metaplastic and neoplastic liver. J Pathol 188:201-206

13. Litvinov SV, Bakker HAM, Gourevitch MM, Velders MP, Warnaar SO (1994) Evidence for a role of the epithelial glycoprotein 40 (Ep-CAM) in epithelial cell-cell adhesion. Cell Adhes Commun 2:417-428

14. Potempa S, Ridley AJ (1998) Activation of both MAP kinase and phosphoinositide 3-kinase by Ras is required for hepatocyte growth factor/scatter factor-induced adherens junction disassembly. Mol Biol Cell 9:2185-2200

15. Braga VM, Betson M, Li X, Lamarche-Vane N (2000) Activation of the small GTPase Rac is sufficient to disrupt cadherin-dependent cell-cell adhesion in normal human keratinocytes. Mol Biol Cell 11:3703-3721

16. Shibasaki F, Fukata M, Fukui Y, Takenawa T (1994) Posphatidylinositol 3-kinase binds to $\alpha$-actinin through the p85 subunit. Biochem J 302:551-557

17. Nelson CM, Chen CS (2002) Cell-cell signaling by direct contact increases cell proliferation via an Pi3K-dependent signal. FEBS Lett 514:238-242

18. Klippel A, Escobedo MA, Wachowicz MS, Apell G, Brown TW, Giedlin MA, Kavanaugh WM, Williams LT (1998) Activation of phosphatidylinositol 3-kinase is sufficient for cell cycle entry and promotes cellular changes characteristic of oncogenic transformation. Mol Cell Biol 18:56995711

19. Grille SJ, Bellacosa A, Upson J, Klein-Szanto AJ, van Roy F, Lee-Kwon W, Donowitz M, Tsichlis PN, Larue L (2003) The protein kinase Akt induces epithelial mesenchymal transition and promotes enhanced motility and invasiveness of squamous cell carcinoma lines. Cancer Res 63:2172-2178

20. Royal I, Fournier TM, Park M (1997) Differntial requirement Grb2 and PI3-kinase in HGF/SF-induced cell motility and tubulogenesis. J Cell Phys 173:196-201

21. Balzar M, Breuer M, Cirulli V, Warnaar SO, Fleuren GJ, Litvinov SV (2001) Ep-CAM is a potent morphoregulatory molecule for mammaryepithelium. In: Thesis "The biology of the epithelial cell adhesion molecule". chapter 7, pp 128-147

22. Keely PJ, Westwick JK, Whitehead IP, Der CJ, Parise LV (1997) Cdc42 and Rac1 induce integrin-mediated cell motility and invasiveness through PI(3)K. Nature 390:632-636

23. Keely PJ, Parise LV, Juliano R (1998) Integrins and GTPases in tumor cell growth, motility and invasion. Trends Cellbiol 8:101-106 
24. Gautreau A, Poullet P, Louvard D, Arpin M (1999) Ezrin, a plasma-microfilament linker, signals through the phosphatidylinositol-3 kinase/Akt pathway. Proc Natl Acad Sci USA 96:7300-7305

25. Perez OD, Kinoshita S, Hitoshi Y, Payan DG, Kitamura T, Nolan GP, Lorens JB (2002) Activation of the PKB/AKT pathway by ICAM-2. Immunity 16:51-65

26. Wennstrom S, Downward J (1999) Role of phosphoinositide 3kinase in activation of ras and mitogen-activated protein kinase by epidermal growth factor. Mol Cell Biol 19:4279-4288

27. Pece S, Chiariello M, Murga C, Gutkind JS (1999) Activation of the protein kinase $\mathrm{Akt} / \mathrm{PKB}$ by the formation of E-cadherin-mediated cell-cell junctions. Evidence for the association of phosphatidylinositol 3-kinase with the E-cadherin adhesion complex. J Biol Chem 274:1934719351

28. Shayesteh L, Lu Y, Kuo WL, Baldocchi R, Godfrey T, Collins C, Pinkel D, Powell B, Mills GB, Gray JW (1999) PIK3CA is implicated as an oncogene in ovarian cancer. Nat Genet 21:99-102

29. Ma YY, Wei SJ, Lin YC, Lung JC, Chang TC, WhangPeng J, Liu JM, Yang DM, Yang WK, Shen CY (2000) PIK3CA as an oncogene in cervical cancer. Oncogene 19:2739-2744 